\title{
OBESIDAD Y AUTORREGULACIÓN DE LA ACTIVIDAD FÍSICA Y LA ALIMENTACIÓN EN ESTUDIANTES UNIVERSITARIOS: UN ESTUDIO LONGITUDINAL
}

\section{OBESITY AND SELF-REGULATION OF PHYSICAL ACTIVITY AND EATING BEHAVIORS IN COLLEGE STUDENTS: A PROSPECTIVE STUDY ${ }^{1}$}

\author{
Yolanda Campos-Uscanga ${ }^{1}$ (0000-0002-5114-3621) \\ Tania Romo-González² (0000-0003-0097-4780) \\ Luis Eduardo del Moral-Trinidad ${ }^{3}$ \\ Nelly Itzel Carmona-Hernández ${ }^{3}$ \\ ${ }^{1}$ Instituto de Salud Pública, Universidad Veracruzana, Xalapa, Veracruz, México \\ 2. Instituto de Investigaciones Biológicas, Universidad Veracruzana, Xalapa, Veracruz, \\ México. \\ ${ }^{3}$ Estudiantes de la Licenciatura en Medicina, Universidad Veracruzana, Xalapa, \\ Veracruz, México \\ ycampos@uv.mx
}

\begin{abstract}
Resumen
El presente estudio tuvo como propósito determinar si existen variaciones en el índice de masa corporal durante los dos primeros años de estudios universitarios y si estas variaciones están influidas por la autorregulación. Se trata de un estudio longitudinal cuantitativo del año 2013 al 2015. Participaron 59 estudiantes de la Licenciatura en Enfermería de una universidad pública de México que ingresaron a esta en el 2013. Se empleó la Escala de autorregulación de hábitos alimentarios y la Escala de autorregulación de la actividad física, además de equipo calibrado para la antropometría. Los resultados mostraron que en el grupo de mujeres el peso, el IMC, la cintura y la cadera aumentaron en la medición final con respecto a la basal, mientras que el ICC $(\mathrm{t}=3.89 ; \mathrm{p}<0.001)$ presentó descenso y la autorregulación se mantuvo sin cambios. Por su parte, en el grupo de hombres se observó aumento en la circunferencia de cintura y de cadera y disminución en la autorregulación de la alimentación. Los valores iniciales en autoevaluación de la actividad física y de la alimentación están asociados con el índice de masa corporal en la última medición $(\beta=0.353 ; p=0.006)$ y mayores puntajes iniciales en autoobservación de la actividad física predicen disminución del IMC $(\beta=$ $0.782 ; \mathrm{p}=0.001$ ). Los hallazgos muestran que menor autorregulación de la actividad física se asocia con aumento del IMC, confirmando la hipótesis de que la autorregulación no solo es útil en el tratamiento, sino también en la prevención de la obesidad.
\end{abstract}

Palabras Claves: índice de masa corporal, actividad física, conducta alimentaria, estudiantes.

\begin{abstract}
This study aims to determinate whether there are variations in the Body Mass Index (BMI) during the first two years of college and if these variations are influenced by self-regulation. This is a quantitative longitudinal study from 2013 to 2015. A total of 59 nursing students admitted in 2013 to a public university in Mexico participated in the study. Self-regulation scales for eating behavior and physical activity were
\end{abstract}


used, in addition to well calibrated anthropometry equipment. Results showed that in females weight, BMI, waist and hip circumference increased compared to the initial data, while the waist/hip ratio $(t=3.89 ; \mathrm{p}$ $<0.001)$ decreased, and self-regulation remained unchanged. On the other hand, in males waist and hip circumference increased, while self-regulation of eating behavior decreased. Initial values in self-evaluation of physical activity and eating behavior are associated with BMI in the last measure $(\beta=0.353 ; p=0.006)$; in addition, higher initial scores in self-observation of physical activity predict a decrease in BMI $(\beta=0.782$; $p=0.001$ ). Findings suggest that lower levels of self-regulation of physical activity are linked to an increase in BMI, which confirms the hypothesis that self-regulation skills are useful not only in treating but also in preventing obesity.

Keywords: body mass index, physical activity, eating behavior, students.

\section{Introducción}

Alrededor del mundo, en el periodo de 1980 a 2013, aumentó la proporción de adultos con exceso de peso en 28.8 \% (Ng et al., 2014) y, en la actualidad, México y Estados Unidos ocupan los primeros lugares de prevalencia de obesidad en adultos (Dávila-Torres, González-Izquierdo y Barrera-Cruz, 2015). Para 2016, la prevalencia combinada de sobrepeso y obesidad en México fue de $72.5 \%$ (Instituto Nacional de Salud Pública, 2016). Este panorama epidemiológico no solo es alarmante al ser la obesidad una condición patológica, sino además porque actualmente los esfuerzos por controlar las enfermedades crónicas no transmisibles ponen especial atención a la obesidad por la asociación que ha mostrado como factor de riesgo (Ogunbode, Ladipo, Ajayi y Fatiregun, 2012). En este sentido, se vuelve fundamental poner en marcha estrategias preventivas y de abordaje de la obesidad.

En su aparición y tratamiento se ha evidenciado la importancia de los comportamientos de los individuos, especialmente los vinculados con la actividad física y la alimentación (Ogunbode et al., 2012). Al respecto del papel del individuo para la prevención y tratamiento de la obesidad, Bandura (1986) ha señalado que es necesario desarrollar ciertas capacidades para la autorregulación del comportamiento que permitan al individuo responder favorablemente ante los estímulos adversos del contexto. La autorregulación hace referencia a procesos internos que se dan en el sujeto con la finalidad de dirigir sus comportamientos hacia el logro de objetivos (Zimmerman, 1989), a través de tres dimensiones: autoobservación, autoevaluación y autorreacción. Estas dimensiones implican la autoobservación de las conductas y el contexto en que se presentan, la autoevaluación de las mismas a través de la comparación con las metas planteadas o estándares ideales y, finalmente, la autorreación para brindar los mecanismos de regulación de acuerdo con los estándares y objetivos establecidos considerando las consecuencias de los comportamientos (Bandura, 1991).

Aunque la teoría de la autorregulación surge en el contexto del aprendizaje, posteriormente se aplica también de forma exitosa a comportamientos vinculados con la salud, incluidos la 
alimentación y la actividad física. Estudios recientes muestran que las personas con obesidad que mejoran su autorregulación tienen buen pronóstico para la pérdida del exceso de peso (Annesi, 2013; Annesi, Johnson y Porter, 2015; Gianessi, 2012; Halberstadt et al., 2017), mientras que una persona con obesidad y baja autorregulación difícilmente logrará mejorar su estado nutricional (Annesi y Porter, 2013; Carraca et al., 2013).

De similar manera, algunos estudios han revelado que la autorregulación presenta valores más bajos en mujeres con obesidad en comparación con quienes tienen peso normal (CamposUscanga, Gutiérrez-Ospina, Morales-Romero y Romo-González, 2016a; Campos-Uscanga, Morales-Ortiz y Argüelles-Nava, 2016b). Lo anterior permite suponer que la autorregulación es un factor clave en la etiología y tratamiento de la obesidad. No obstante, se carece de evidencia sobre si dicha asociación es causal ya que no existen estudios longitudinales que permitan determinar si la autorregulación antecede a la obesidad y pudiera incluso considerarse como un factor protector. Por lo que, si bien hay evidencia que en personas con obesidad la autorregulación pronostica éxito en la pérdida de peso, no hay evidencia de lo que sucede en personas con peso normal.

Asimismo, existe evidencia que apunta a que no todas las dimensiones de la autorregulación favorecen un adecuado estado nutricio. Por ejemplo, en un estudio previo se observó que la autoevaluación de la alimentación se comporta como factor de riesgo para la presencia de obesidad (Campos-Uscanga et al., 2016a); sin embargo, no es claro el motivo por el cual se da este tipo de asociación, la cual incluso pudiera deberse al uso de inadecuados estándares para evaluar los comportamientos.

Tal como se mostró previamente, México no solo ocupa los primeros lugares en obesidad a nivel mundial sino también esta prevalencia se presenta a edades cada vez más tempranas. Al respecto, el periodo de estudios universitarios implica cambios importantes en los estilos de vida por lo que es una etapa crítica para la ganancia de peso corporal y la adquisición de conductas poco saludables (Baum, 2016; Laska, Pelletier, Larson y Story, 2012), aunque también puede ser un área de oportunidad para el desarrollo de habilidades de autorregulación que den lugar a la consolidación de estilos de vida saludable (Arnett y Padilla-Walker, 2015; Baum, 2016). Sin embargo, esta evidencia sobre la relevancia de los comportamientos ha dado lugar a algunas malinterpretaciones que demeritan la responsabilidad del sistema de salud, las instituciones y la industria (Rust, 2017; Tempels, Verweij y Blok, 2017). Debe tenerse presente que como seres sociales y parte de un sistema los contextos tienen gran influencia en los comportamientos, de otra forma parecería que el individuo fuera un ente aislado que actúa sin ser influido por la realidad social (Matheson, 2016). Esta realidad cada vez deja menos tiempo para el cuidado de la salud, con jornadas laborales y escolares extenuantes; inseguridad en los ambientes comunitarios que limitan las actividades al aire libre; inequidades económicas que limitan el acceso a comida saludable; entre otros más.

La universidad como institución debería ser una auténtica promotora de la salud y generar las condiciones para que se arraiguen comportamientos que favorezcan la salud; sin embargo, 
este trabajo es apenas incipiente (Baum, 2016; Partridge, Juan, McGeechan, Bauman y AllmanFarinelli, 2015). En este sentido hay evidencia controvertida, ya que por un lado algunos autores señalan que durante el periodo universitario los estudiantes tienden a ganar peso más allá de lo saludable, mientras que otros autores aseguran que esta ganancia es solo transitoria. Baum señala que durante el primer año de estudios universitarios, los jóvenes ganan 1.8 kilogramos en promedio y de 900 a 1300 gramos en los años siguientes, pero medias similares se observan en los jóvenes que no asisten a la universidad (Baum, 2016). Lo anterior indica que la asistencia a la universidad por sí sola no lleva al aumento de peso, de hecho, los egresados de las universidades tienen menos probabilidad de tener obesidad en el futuro que los que no estudiaron (Baum, 2016). Bajo esta evidencia, queda explícito que la educación pudiera ser un factor protector de obesidad. No obstante, otros estudios señalan que durante el periodo de estudios universitarios hay ganancia significativa de peso corporal y aumenta la prevalencia de exceso de peso (Gropper, Simmons, Connell y Ulrich, 2012; Pope, Hansen y Harvey, 2016).

Por lo expuesto anteriormente, el objetivo de este trabajo fue determinar si existen variaciones en el índice de masa corporal durante los dos primeros años de estudios universitarios y si estas variaciones están influidas por la autorregulación. La hipótesis es que durante los dos primeros años de estudios universitarios los estudiantes ganan peso corporal de forma significativa, lo cual se explica por los bajos niveles de autorregulación de la actividad física y la alimentación.

\section{Metodología}

\section{Participantes}

Se realizó un estudio longitudinal cuantitativo del año 2013 al 2015. Participaron estudiantes de la Facultad de Enfermería Región Veracruz, de la Universidad Veracruzana, que ingresaron a esta en el 2013. La media de edad al inicio del estudio fue de $18.7(\mathrm{DE}=0.9)$, con un mínimo de 17 y máximo de 21.

Inicialmente se incluyeron en el estudio 100 estudiantes, sin embargo, fueron excluidos 41 por no completar los dos años de seguimiento (10 por deserción y 31 por no encontrarse en la Universidad al momento del levantamiento de datos), quedando con una muestra final de 59 participantes, de los cuales 41 fueron mujeres y 18 fueron hombres, lo que corresponde a la distribución por sexo que existe en la matrícula de la carrera de Enfermería en la Universidad Veracruzana.

\section{Instrumentos}

Escala de autorregulación de hábitos alimentarios. Fue desarrollada por CamposUscanga, Lagunes, Morales-Romero y Romo-González (2015). Consta de 14 reactivos en escala tipo Likert con cinco opciones de respuesta que van de "nunca" a "siempre" y están agrupados 
en tres dimensiones. La calificación total de la escala va de 14 a 70 puntos considerando que a mayor puntaje, mejor autorregulación. Los factores se integran de la siguiente manera: Autoobservación, cuatro reactivos $(\boldsymbol{\alpha}=0.730)$; Autoevaluación, cuatro reactivos $(\boldsymbol{\alpha}=0.719)$; Autorreacción, seis reactivos $(\boldsymbol{\alpha}=0.864)$. La escala explica $46.9 \%$ de la varianza y la confiabilidad es adecuada para la prueba general $(\boldsymbol{\alpha}=0.874)$ (Campos-Uscanga et. al., 2015).

Escala de autorregulación de la actividad física. Fue desarrollada por Campos-Uscanga, Lagunes, Morales-Romero y Romo-González (2016c). Consta de 12 reactivos en escala tipo Likert con cinco opciones de respuesta que van de "nunca" a "siempre" y están agrupados en tres dimensiones. La calificación total de la escala va de 12 a 60 puntos considerando que a mayor puntaje, mejor autorregulación. Los factores se integran de la siguiente manera: Autoobservación, cinco reactivos $(\boldsymbol{\alpha}=0.744)$; Autoevaluación, dos reactivos $(\boldsymbol{\alpha}=0.686)$; Autorreacción, cinco reactivos $(\boldsymbol{\alpha}=0.824)$. La escala explica $49.4 \%$ de la varianza y la confiabilidad es adecuada para la prueba general $(\boldsymbol{\alpha}=0.846)$ (Campos-Uscanga, et. al., 2016c).

Además, se preguntó sobre algunas variables como la edad, género, realizar alguna actividad física programada y las horas que le dedica a la semana a dicha actividad.

Para la medición de peso corporal se empleó una báscula de precisión marca Taylor, calibrada previamente; para la medición de la talla se utilizó un estadímetro de precisión marca Seca ${ }^{\circledR}$ y para la medición de circunferencias se utilizó una cinta graduada flexible e inextensible de fibra de vidrio marca Seca®.

\section{Procedimiento}

Previo al inicio del estudio, el protocolo fue registrado y aprobado por el Comité de ética de la institución sede del trabajo; además, los procedimientos realizados se apegaron a la Declaración de Helsinki. Los participantes lo hicieron de forma voluntaria, firmando un consentimiento informado, y se protegió en todo momento su anonimato.

La medición de autorregulación y la antropometría fueron realizados el mismo día, una vez que los estudiantes hacían el llenado de las escalas pasaban a un espacio privado para la antropometría, que fue realizada por nutriólogos entrenados. El sujeto fue pesado con el mínimo de ropa posible, descalzo y con evacuación de vejiga urinaria. Posteriormente, para la medición de la talla se mantuvieron descalzos y sin adornos en la cabeza. La circunferencia de cintura se tomó sobre el torso descubierto ubicando el punto medio del borde costal y la cresta iliaca, y verificando que el abdomen se encontrara relajado. La medición de la circunferencia de cadera se efectuó localizando el nivel de la sínfisis púbica y el punto más prominente de la región glútea.

\section{Análisis estadísticos}

Para el análisis de los datos se utilizó el software SPSS Inc. Released 2009. PASW Statistics for Windows, Version 18.0. Chicago: SPSS Inc. Para determinar la normalidad de los 
datos, se empleó la asimetría y curtosis, identificando que todas las variables de autorregulación y antropometría seguían una distribución normal, por lo que fueron presentadas a través de medias y desviación estándar. Posteriormente, se hicieron comparaciones entre la medición inicial y la final a través de la $t$ de student. La comparación se hizo por géneros debido a las diferencias en composición corporal y puntos de corte que existen entre hombres y mujeres y el desbalance en el tamaño de los grupos que pudo haber sesgado los resultados. Se empleó la correlación de Pearson para identificar asociaciones entre antropometría, autorregulación y horas que dedican a la actividad física. Finalmente, se realizaron dos análisis multivariados a través de regresión lineal teniendo como variables dependientes para el primer modelo el IMC en la medición final y para el segundo modelo las diferencias en el IMC entre la medición basal y la final. En dichos modelos se incluyeron las variables de autorregulación, edad, horas que realiza actividad física e IMC inicial. Se revisó que cumplieran el resto de los supuestos a través puntaje de la prueba de Durbin-Watson, que indicó independencia de errores (2.359), y el factor de inflación de la varianza, que indicó la no multicolinealidad (valores entre 1.268 y 3.470). Para todos los análisis se identificaron como significativos los valores de $\mathrm{p}$ inferiores a 0.05 .

\section{Resultados}

Desde la medición basal hasta los 2 años de seguimiento en el grupo de mujeres las variables de peso, IMC, cintura y cadera aumentaron, mientras que el ICC presentó un descenso significativo. En el grupo de hombres, se observó un aumento en la media de circunferencia de cintura y de cadera, mientras que las demás variables no presentaron cambios significativos (Tabla 1).

Tabla 1

Comparación de medias de antropometría entre la medición basal y la de seguimiento por sexo

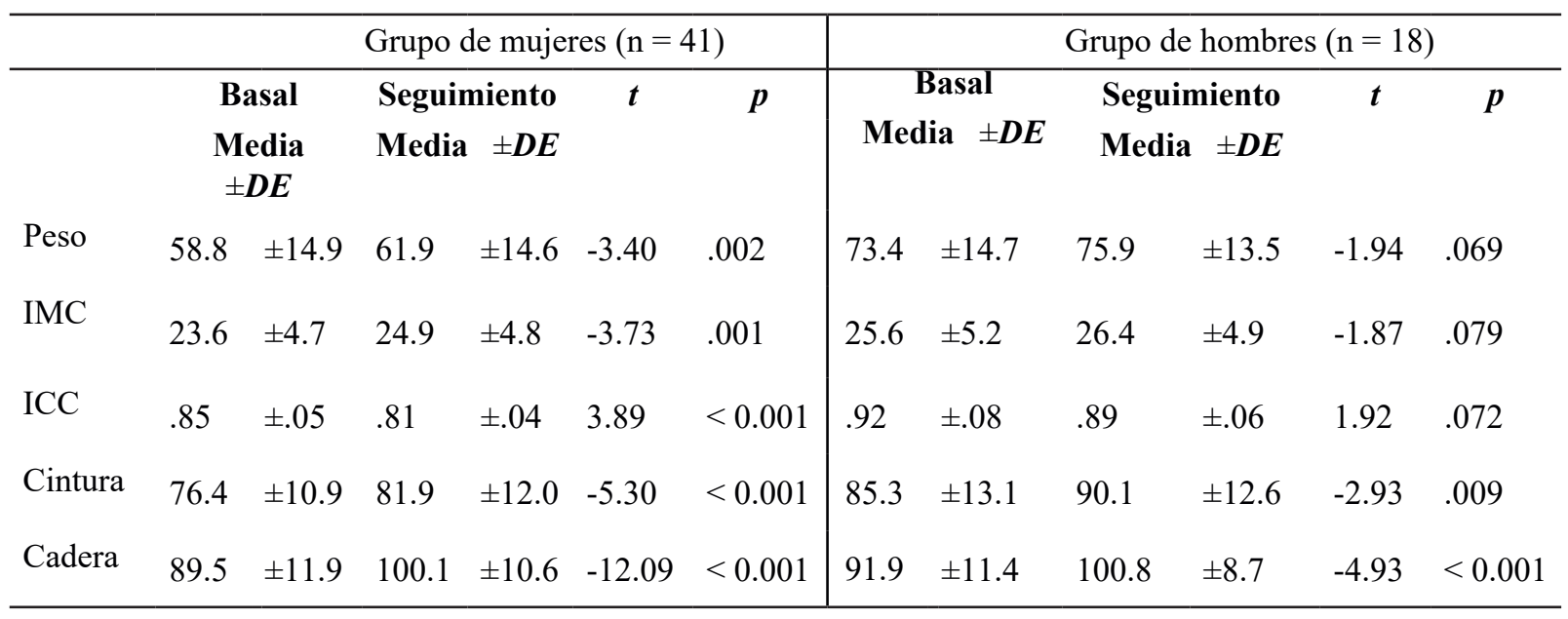


No hubo modificación en la autorregulación entre la medición inicial y tras dos años de seguimiento en el grupo de mujeres; mientras que en el grupo de hombres la media de autorregulación de alimentación disminuyó significativamente (Tabla 2).

Tabla 2.

Comparación de medias de autorregulación en la medición basal y de seguimiento en la muestra

\begin{tabular}{|c|c|c|c|c|c|c|c|c|c|c|c|c|}
\hline \multirow[b]{4}{*}{ Alimentación } & \multicolumn{6}{|c|}{ Grupo de mujeres $(n=41)$} & \multicolumn{6}{|c|}{ Grupo de hombres $(n=18)$} \\
\hline & \multirow{2}{*}{\multicolumn{2}{|c|}{$\begin{array}{c}\text { Basal } \\
\text { Media } \\
\pm D E\end{array}$}} & \multirow{2}{*}{\multicolumn{2}{|c|}{$\begin{array}{c}\text { Segui- } \\
\text { miento } \\
\text { Media } \\
\pm D E\end{array}$}} & \multirow[t]{3}{*}{$t$} & \multirow[t]{3}{*}{$p$} & \multicolumn{2}{|c|}{ Basal } & \multicolumn{2}{|c|}{ Seguimiento } & \multirow[t]{2}{*}{$t$} & \multirow[t]{3}{*}{$P$} \\
\hline & & & & & & & \multirow{2}{*}{\multicolumn{2}{|c|}{ Media $\pm D E$}} & \multirow{2}{*}{\multicolumn{2}{|c|}{ Media $\pm D E$}} & & \\
\hline & & & & & & & & & & & & \\
\hline Autorregulación & 45.2 & \pm 7.9 & 47.2 & \pm 8.1 & -1.32 & .193 & 45.8 & \pm 7.4 & 41.4 & \pm 10.9 & 2.24 & $.038^{*}$ \\
\hline Autoobservación & 16.4 & \pm 2.6 & 17.0 & \pm 2.5 & -1.35 & .185 & 16.1 & \pm 2.5 & 14.9 & \pm 3.4 & 1.51 & .150 \\
\hline Autoevaluación & 11.5 & \pm 3.2 & 11.8 & \pm 3.1 & -0.58 & .565 & 12.0 & \pm 2.7 & 10.6 & \pm 3.8 & 1.73 & .103 \\
\hline Autorreacción & 17.2 & \pm 4.8 & 18.3 & \pm 4.8 & -1.27 & .213 & 17.8 & \pm 4.4 & 15.9 & \pm 4.9 & 2.05 & .056 \\
\hline \multicolumn{13}{|l|}{ Actividad física } \\
\hline Autorregulación & 42.9 & \pm 6.4 & 42.6 & \pm 7.2 & 0.27 & .787 & 43.2 & \pm 8.0 & 41.3 & \pm 10.2 & 1.15 & .266 \\
\hline Autoobservación & 20.3 & \pm 3.0 & 19.7 & \pm 3.6 & 0.88 & .383 & 19.7 & \pm 3.4 & 18.4 & \pm 4.0 & 1.46 & .164 \\
\hline Autoevaluación & 8.3 & \pm 1.7 & 8.1 & \pm 1.7 & 0.87 & .391 & 7.4 & \pm 1.8 & 7.6 & \pm 2.1 & -0.24 & .816 \\
\hline Autorreacción & 14.3 & \pm 4.1 & 14.8 & \pm 3.9 & -0.71 & .484 & 16.0 & \pm 4.1 & 15.3 & \pm 4.8 & 1.23 & .236 \\
\hline
\end{tabular}

Se encontraron distintas correlaciones inversas significativas: edad con autorregulación $(\mathrm{r}=-.258 ; \mathrm{p}=.048)$ y autorreacción $(\mathrm{r}=-.267 ; \mathrm{p}=.041)$ de la actividad física; circunferencia de cintura con autoobservación de la alimentación $(\mathrm{r}=-.256 ; \mathrm{p}=.049)$ y autoevaluación de la actividad física $(\mathrm{r}=-.386 ; \mathrm{p}=.003)$; circunferencia de cadera con autoobservación de la alimentación $(\mathrm{r}=-.319 ; \mathrm{p}=.014)$ autorreacción de la alimentación $(\mathrm{r}=-0.262 ; \mathrm{p}=0.045)$ y autoevaluación de la actividad física $(\mathrm{r}=-.416 ; \mathrm{p}=.001)$. Finalmente, también se encontró el índice de masa corporal con la autoobservación de la alimentación $(\mathrm{r}=-.327 ; \mathrm{p}=.011)$ y la autoevaluación de la actividad física $(\mathrm{r}=-.461 ; \mathrm{p}<.001)$ (Tabla 3). 
Tabla 3.

Correlaciones entre autorregulación y antropometría

\begin{tabular}{|c|c|c|c|c|c|c|}
\hline & Edad & $\begin{array}{c}\text { Horas de } \\
\text { actividad } \\
\text { física } \\
\end{array}$ & $\begin{array}{l}\text { Circunferencia } \\
\text { de cintura }\end{array}$ & $\begin{array}{l}\text { Circunferencia } \\
\text { de cadera }\end{array}$ & $\begin{array}{c}\text { Índice } \\
\text { de masa } \\
\text { corporal }\end{array}$ & $\begin{array}{c}\text { Índice de cintura/ } \\
\text { cadera }\end{array}$ \\
\hline \multicolumn{7}{|l|}{ Alimentación } \\
\hline Autorregulación & .076 & -.069 & -.186 & -.213 & -.228 & -.012 \\
\hline Autoobservación & .126 & -.162 & $-.256^{*}$ & $-.319^{*}$ & $-.327 *$ & .016 \\
\hline Autoevaluación & .071 & -.053 & .090 & .127 & .086 & -.026 \\
\hline \multirow[t]{2}{*}{ Autorreacción } & .010 & .003 & -.227 & $-.262 *$ & -.255 & -.011 \\
\hline & Edad & $\begin{array}{c}\text { Horas de } \\
\text { actividad } \\
\text { física } \\
\end{array}$ & $\begin{array}{l}\text { Circunferencia } \\
\text { de cintura }\end{array}$ & $\begin{array}{l}\text { Circunferencia } \\
\text { de cadera }\end{array}$ & $\begin{array}{c}\text { Índice } \\
\text { de masa } \\
\text { corporal }\end{array}$ & $\begin{array}{c}\text { Índice de cintura/ } \\
\text { cadera }\end{array}$ \\
\hline \multicolumn{7}{|l|}{ Actividad física } \\
\hline Autorregulación & $-.258^{*}$ & -.067 & -.153 & -.142 & -.187 & -.049 \\
\hline Autoobservación & -.113 & -.020 & -.049 & .032 & 0.010 & -.129 \\
\hline Autoevaluación & -.180 & -.170 & $-.386^{* *}$ & $-.416^{* *}$ & $-.461 * *$ & -.068 \\
\hline Autorreacción & $-.267 *$ & -.023 & .055 & -.085 & -.123 & .043 \\
\hline
\end{tabular}

Nota: Coeficientes de correlación de Pearson, ${ }^{\star} \mathrm{p}<0.05 ;{ }^{\star *} \mathrm{p}<0.01$

En el primer modelo se encontró que los valores iniciales en autoevaluación de la actividad física y de la alimentación están asociados con el índice de masa corporal al final del estudio (Tabla 4).

Tabla 4

Análisis multivariado para índice de masa corporal en la medición final

\begin{tabular}{lccccc}
\hline \multicolumn{1}{c}{ Variables en la medición inicial } & $\mathbf{B}$ & $\begin{array}{c}\text { Error } \\
\text { estándar }\end{array}$ & $\boldsymbol{\beta}$ & $\mathbf{t}$ & $\mathbf{p}$ \\
\hline Constante & 19.014 & 10.869 & & 1.749 & .101 \\
IMC & .921 & .097 & 1.065 & 9.447 & $<0.001$ \\
Edad & -.932 & .535 & -.153 & -1.741 & .102 \\
Horas que realiza actividad física & -.052 & .077 & -.057 & -.674 & .511 \\
Autoobservación de la alimentación & -.091 & .196 & -.045 & -.465 & .649 \\
Autoevaluación de la alimentación & .307 & .140 & .186 & 2.189 & .045 \\
Autorreacción de la alimentación & -.049 & .103 & -.050 & -.481 & .637 \\
Autoobservación de actividad física & -.296 & .216 & -.191 & -1.371 & .191 \\
Autoevaluación de actividad física & .810 & .252 & .353 & 3.220 & .006 \\
Autorreacción de actividad física & -.030 & .170 & -.024 & -.176 & .863 \\
\hline
\end{tabular}

Nota: $R^{2}=0.866$ 
En el segundo modelo la autoobservación de la actividad física muestra asociación inversa con la disminución del IMC, mientras que la autoevaluación de la actividad física también se asoció con la disminución del IMC pero de forma positiva (Tabla 5 y Figura 2).

Tabla 5.

Medidas basales como predictoras de cambio en el índice de masa corporal

\begin{tabular}{|l|c|c|c|c|c|}
\hline \multicolumn{1}{|c|}{ Variables en la medición inicial } & $B$ & $\begin{array}{c}\text { Error } \\
\text { estándar }\end{array}$ & $\beta$ & $t$ & $p$ \\
Constante & 19.531 & 10.735 & & 1.819 & .088 \\
Edad & -1.022 & .518 & -.330 & -1.973 & .066 \\
Horas que realiza actividad física & -.071 & .072 & -.153 & -.978 & .343 \\
Autoobservación de la alimentación & -.107 & .193 & -.104 & -.555 & .587 \\
Autoevaluación de la alimentación & .277 & .134 & .330 & 2.069 & .055 \\
Autorreacción de la alimentación & -.045 & .101 & -.089 & -.440 & .666 \\
Autoobservación de actividad física & -.396 & .175 & -.503 & -2.259 & .038 \\
Autoevaluación de actividad física & .910 & .217 & .782 & 4.194 & .001 \\
Autorreacción de actividad física & .037 & .147 & .060 & .254 & .803 \\
\hline
\end{tabular}

Nota: $\mathrm{R}^{2}=0.480$

Un parámetro negativo indica que su incremento predice mayor pérdida de IMC (pérdida de IMC es una puntuación negativa de cambio)

Se identificó que mayores puntajes iniciales en autoobservación de la actividad física predicen disminución del IMC (Fig. 1), mientras que mayores puntajes en autoevaluación de la actividad física predicen ganancia de IMC (Fig. 2).



Figura 1. Regresión parcial: diferencia en el índice de masa corporal por autoobservación de la actividad física 


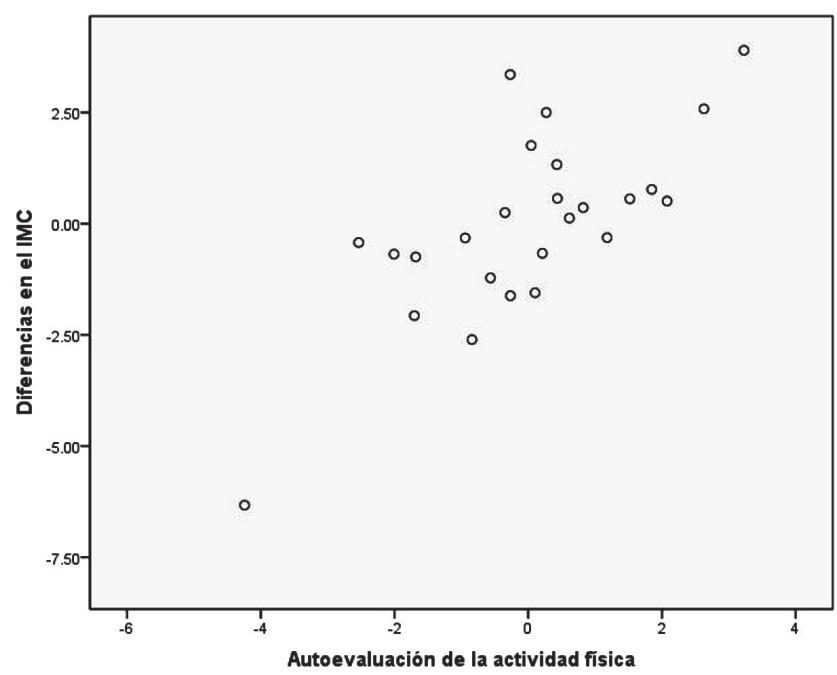

Figura 2. Regresión parcial: diferencia en el índice de masa corporal por autoevaluación de la actividad física

\section{Discusión}

El periodo de estudios universitarios hace coincidir a una etapa del desarrollo en la cual los jóvenes consolidan estilos de vida (Arnett y Padilla-Walker, 2015) con la adquisición de habilidades y conocimientos para el ejercicio de una profesión. Se espera que los estudiantes de las áreas de la salud evidencien comportamientos coherentes con su formación, especialmente aquellos cuya labor se dirige justamente hacia el cuidado y la promoción de la salud (Salehi et al., 2017). Por lo anterior, las universidades deben ser formadoras integrales que superen el ámbito de los conocimientos para influir en el bienestar de su comunidad estudiantil.

Si bien la misión de la universidad en la promoción de la salud es clara, diversos indicadores muestran que se está fallando en estas instancias. Ejemplo de ello son los altos índices en obesidad que la población joven en México alcanza (Instituto Nacional de Salud Pública, 2016). Por esto, elaborar estrategias para mejorar estos indicadores son de la más alta prioridad. Diferentes estudios han mostrado que la autorregulación del comportamiento es la estrategia más viable dentro de los programas de reducción del exceso de peso pero la investigación con enfoque preventivo es limitada por lo que es necesario tener evidencia longitudinal sobre el valor de este constructo para la puesta en marcha de programas para la promoción de la salud.

En el presente estudio se observó que el peso corporal, el IMC, la circunferencia de cintura y la circunferencia de cadera tuvieron aumentos importantes a lo largo de los dos años de seguimiento en el grupo de mujeres. Si bien en este grupo el ICC disminuyó, esto se debe al aumento tan drástico en la circunferencia de cadera que se observó en la muestra señalando 
una acumulación de tejido en esa región, ya que uno de los indicadores para estimar el ICC es justamente la circunferencia de cadera (Gateva y Kamenov, 2012). Aunque no puede afirmarse que el tejido desarrollado sea graso, puede sospecharse por la complexión típica de la mujer veracruzana. Estos resultados son similares en el grupo de hombres en los que hubo un aumento significativo en las circunferencias de cintura y cadera. Estos resultados coinciden con estudios previos donde se identificó que, ante la ausencia de intervenciones para regular el peso, los estudiantes tienden a ganar peso corporal más allá de lo saludable (Campos-Uscanga, Argüelles, Vázquez y Ortiz, 2014). Es decir, estos resultados muestran que cuando las instituciones educativas no asumen su responsabilidad en la promoción de la salud, se mantienen o adquieren conductas poco saludables por las limitadas capacidades para regular los comportamientos.

Por otra parte, la permanencia en los puntajes de autorregulación apunta a que pese a aumentar sus conocimientos sobre salud y cuidado, como se espera de la formación profesional, esto no resulta suficiente para que generen cambios en su capacidad para regular comportamientos. Incluso, en el grupo de hombres hubo disminución significativa en la capacidad de autorregulación de la alimentación. Al parecer la universidad no solo no está fomentando su desarrollo, sino que, como lo señalaban Partridge et al. (2015) en algunos casos se presentan retrocesos y se deja de cumplir la responsabilidad como promotora de la salud

Al respecto, los hallazgos de esta investigación muestran que aquellos indicadores de obesidad como el índice de masa corporal, la circunferencia de cintura y la circunferencia de cadera correlacionaron de forma inversa con la autoobservación de la alimentación y la autoevaluación de la actividad física. Aunque investigaciones previas habían dado ya acercamientos a esta asociación por los efectos que las intervenciones dirigidas a la pérdida de peso habían mostrado (Annesi, 2013; Annesi, Johnson y Porter, 2015; Gianessi, 2012; Halberstadt et al., 2017), en el presente estudio fue además posible asociarlos con estos índices antropométricos que han sido identificados como los más fuertemente relacionados a enfermedades crónicas como la hipertensión (Janghorbani, Aminorroaya y Amini, 2017). Así mismo, estos resultados muestran que dentro de la autorregulación se debe prestar atención a sus distintas dimensiones, pues el estudiar solo el proceso global puede no identificar algunas relaciones o enmascararlas, perdiendo fidelidad en la determinación de estos elementos como fundamentales a fortalecer en los jóvenes.

De similar manera, la autorreacción de la alimentación mostró estar relacionada con la circunferencia de cadera, mientras que un estudio previo la había mostrado asociada a la circunferencia de cintura (Campos-Uscanga et. al., 2016a). Si se tiene en cuenta que la autorreacción implica la puesta en marcha y seguimiento de un plan para alcanzar objetivos autodeterminados (Bandura, 1991), es de esperarse que haya una influencia en las circunferencias por hacer referencia a acciones concretas de cambio.

La autoobservación de la actividad física mostró ser importante predictora de variaciones en el IMC. Conforme los estudiantes tenían mejores puntajes en esta dimensión de la 
autorregulación, mostraban mayor pérdida de IMC. El trabajo de Campos-Uscanga, MoralesOrtiz et. al., (2016b) con estudiantes universitarias indígenas ya había señalado a la autoevaluación de la actividad física como factor protector de obesidad.

En suma, estos resultados señalan una dirección para el desarrollo de intervenciones dirigidas a la prevención de la obesidad. Es decir, la autorregulación de la actividad física pudiera ser el punto de partida al que se anclen las acciones de promoción de estilos de vida más saludables en las universidades y el motor para el desarrollo de estrategias que permitan a los jóvenes ser conscientes de sus conductas y las condiciones en que se dan. Yendo aún más allá, estos resultados permiten suponer que los aportes de la actividad física no se quedan en el terreno de la salud física y pudieran estar haciendo aportes al bienestar psicológico.

Si bien los hallazgos hasta ahora descritos comprueban la hipótesis de investigación, hubo otros que resultan controvertidos como es el caso de la identificación en ambos modelos multivariados de que la autoevaluación se asoció de forma inversa con el IMC en la medición final y el cambio en IMC. Generalmente se ha visualizado a la autorregulación como factor que protege de la obesidad, sin embargo, en un estudio previo se había hecho patente que la autoevaluación no siempre favorece un adecuado estado nutricio (Campos-Uscanga, et al., 2016a). Esto puede deberse a que los jóvenes no cuenten con estándares adecuados de autoevaluación o que simplemente se enfoquen en la comparación sin dar el paso a la acción. Por esto, se requiere realizar estudios más detallados que permitan determinar los parámetros de evaluación que están usando los jóvenes. Asimismo, no debe perderse de vista que actualmente los medios ejercen una presión muy fuerte sobre los ideales de alimentación y estética que no siempre son acordes con la salud (Dakanalis et al., 2015; Hatfield, Sliwa, Folta, Economos y Goldberg, 2016; Walker et al., 2015).

También resulta necesario realizar estudios longitudinales hasta el final de la formación profesional y en etapas posteriores que permitan identificar variaciones en la autorregulación y el estado nutricio. Sería adecuada además la inclusión de una cohorte no expuesta para valorar si los cambios identificados tienen que ver con la formación universitaria o es parte del ciclo de vida, como han señalado estudios previos (Baum, 2016).

Finalmente, los resultados deben tomarse con reservas debido a la proporción de pérdidas que hubo de participantes a lo largo del estudio. Es posible que esa gran cantidad de estudiantes que no acudieron a la segunda medición en caso de haber participado hubieran aportado elementos distintos a los aquí reportados. También un tamaño de muestra mayor permitiría hacer los análisis multivariados por sexo debido a las diferencias en la composición corporal que pueden influir en las asociaciones encontradas.

\section{Conclusiones}

La autorregulación no solo es una estrategia efectiva en el tratamiento de la obesidad, los hallazgos muestran que también permite prevenir su aparición al hacer evidente que mayor

Revista MHSalud® (ISSN: 1659-097X) Vol. 14. No. 1. Setiembre-Enero, 2017 
autorregulación de la actividad física se asocia con la disminución del IMC, lo que permite confirmar la relación causa-efecto. Sin embargo, el paso por la universidad no está apoyando al fortalecimiento de estas capacidades de autorregulación y en cambio deteriora el estado nutricio. En la medida en que las universidades se involucren con la formación integral de sus estudiantes estaremos en condiciones de ofrecer alternativas que garanticen el impacto social de los profesionales de la salud haciendo congruente el discurso con su actuar. Al tiempo que se fomentan estrategias para el cambio en los individuos, se requiere la generación, desde las instituciones y el sistema de salud, de las condiciones necesarias para que estos cambios se den.

Fecha de recibo:20 de abril del 2017

Fecha de corrección: 13 de junio del 2017

Fecha de aceptación: 27 de julio del 2017

Fecha de publicación: 11 de agosto del 2017

\section{Referencias bibliográficas}

Annesi, J. (2013). Effects of treatment differences on psychosocial predictors of exercise and improved eating in obese, middle-age adults. Journal of Physical Activity and Health, 10(7), 1024-1031. https://doi.org/10.1123/jpah.10.7.1024

Annesi, J., \& Porter, K. (2013). Self-Regulatory Skills for Controlled Eating Emanating From Newly Initiated Physical Activity. Journal of Social, Behavioral, and Health Sciences, 7(1), 67-78. https://doi.org/10.5590/JSBHS.2013.07.1.05

Annesi, J. J., Johnson, P. H., \& Porter, K. J. (2015). Bi-Directional Relationship Between Self-Regulation and Improved Eating: Temporal Associations With Exercise, Reduced Fatigue, and Weight Loss. The Journal of Psychology, 149(6), 535-553. https://doi.org/10.1080/00223980.2014.913000

Arnett, J. J., \& Padilla-Walker, L. M. (2015). Brief report: Danish emerging adults' conceptions of adulthood. Journal of Adolescence, 38, 39-44. https://doi.org/10.1016/j. adolescence.2014.10.011

Bandura, A. (1986). Social foundations of thought and action: A social cognitive theory. New Jersey, EE. UU.: Prentice Hall.

Bandura, A. (1991). Social Cognitive Theory of Self-Regulation. Organizational Behavior and Human Decision Processes, 50(2), 248-287. https://doi.org/10.1016/0749-5978(91)90022-L

Baum, C. L., 2nd. (2016). The Effects of College on Weight: Examining the "Freshman 15" Myth and Other Effects of College Over the Life Cycle. Demography. https://doi.org/10.1007/ $\underline{\text { s13524-016-0530-6 }}$ 
Campos-Uscanga, Y., Argüelles, V., Vázquez, F., \& Ortiz, M. (2014). Autorregulación, autoeficacia y orientación nutricional para la disminución del exceso de peso. Revista Argentina de Clínica Psicológica, 23(2).

Campos-Uscanga, Y., Lagunes, R., Morales-Romero, J., \& Romo-Gonzalez, T. (2015). Diseño y validación de una escala para valorar la autorregulación de hábitos alimentarios en estudiantes universitarios mexicanos. Archivos Latinoamericanos de Nutrición, 65(1), 44-50.

Campos-Uscanga, Y., Gutierrez-Ospina, G., Morales-Romero, J., \& Romo-Gonzalez, T. (2016a). Self-regulation of eating and physical activity is lower in obese female college students as compared to their normal weight counterparts. Eating and Weight Disorders. doi: 10.1007/ s40519-016-0338-9. https://doi.org/10.1007/s40519-016-0338-9

Campos-Uscanga, Y., Morales-Ortiz, A., \& Argüelles-Nava, v. (2016b). Obesity in female student: ethnicity and self-regulation as predicting factors. Health Behavior and Policy Review, 3(4), 315-322. https://doi.org/10.14485/HBPR.3.4.3

Campos-Uscanga, Y., Lagunes, R., Morales-Romero, J., \& Romo-Gonzalez, T. (2016c). Diseño y validación de una Escala de autorregulación de la actividad física. Revista Psicología del Deporte, 25(2), 309-316.

Carraca, E. V., Silva, M. N., Coutinho, S. R., Vieira, P. N., Minderico, C. S., Sardinha, L. B., \& Teixeira, P. J. (2013). The association between physical activity and eating self-regulation in overweight and obese women. Obesity Facts, 6(6), 493-506. https://doi.org/10.1159/000356449

Dakanalis, A., Carra, G., Calogero, R., Fida, R., Clerici, M., Zanetti, M. A., \& Riva, G. (2015). The developmental effects of media-ideal internalization and self-objectification processes on adolescents' negative body-feelings, dietary restraint, and binge eating. European Child \& Adolescent Psychiatry, 24(8), 997-1010. https://doi.org/10.1007/s00787-014-0649-1

Davila-Torres, J., Gonzalez-Izquierdo, J. J., \& Barrera-Cruz, A. (2015). Obesidad en México. Revista Médica del Instituto Mexicano del Seguro Social, 53(2), 240-249.

Gateva, A., \& Kamenov, Z. (2012). Cardiovascular Risk Factors in Bulgarian Patients with Polycystic Ovary Syndrome and/or Obesity. Obstetrics and Gynecology International, 306347. https://doi.org/10.1155/2012/306347

Gianessi, C. A. (2012). From habits to self-regulation: how do we change? [Congresses]. Yale Journal of Biology and Medicine, 85(2), 293-299.

Gropper, S. S., Simmons, K. P., Connell, L. J., \& Ulrich, P. V. (2012). Changes in body weight, composition, and shape: a 4-year study of college students. Applied Physiology, Nutrition and Metabolism, 37(6), 1118-1123. https://doi.org/10.1139/h2012-139 
Halberstadt, J., de Vet, E., Nederkoorn, C., Jansen, A., van Weelden, O. H., Eekhout, I., . . . Seidell, J. C. (2017). The association of self-regulation with weight loss maintenance after an intensive combined lifestyle intervention for children and adolescents with severe obesity. BMC Obesity, 4, 13. https://doi.org/10.1186/s40608-016-0140-2

Hatfield, D. P., Sliwa, S. A., Folta, S. C., Economos, C. D., \& Goldberg, J. P. (2016). The critical role of communications in a multilevel obesity-prevention intervention: Lessons learned for alcohol educators. Patient Education and Counseling. https://doi.org/10.1016/j.pec.2016.01.005

Instituto Nacional de Salud Pública. (2016). Encuesta Nacional de Salud y Nutrición de Medio Camino (pp. 149). México: Secretaría de Salud.

Janghorbani, M., Aminorroaya, A., \& Amini, M. (2017). Comparison of Different Obesity Indices for Predicting Incident Hypertension. High Blood \& Pressure Cardiovascular Prevention, 24(2), 157-166. https://doi.org/10.1007/s40292-017-0186-3

Laska, M. N., Pelletier, J. E., Larson, N. I., \& Story, M. (2012). Interventions for weight gain prevention during the transition to young adulthood: a review of the literature. Journal of Adolescent Health, 50(4), 324-333. https://doi.org/10.1016/j.jadohealth.2012.01.016

Matheson, A. (2016). Reducing social inequalities in obesity: complexity and power relationships. Journal of Public Health (Oxf), 38(4), 826-829. https://doi.org/10.1093/pubmed/fdv197

Ng, M., Fleming, T., Robinson, M., Thomson, B., Graetz, N., Margono, C., . . . Gakidou, E. (2014). Global, regional, and national prevalence of overweight and obesity in children and adults during 1980-2013: a systematic analysis for the Global Burden of Disease Study 2013. Lancet, 384(9945), 766-781. https://doi.org/10.1016/S0140-6736(14)60460-8

Ogunbode, A. M., Ladipo, M., Ajayi, I. O., \& Fatiregun, A. A. (2012). Obesity: An emerging disease. Nigerian Journal of Clinical Practice, 14(4), 390-394. https://doi.org/10.4103/1119$\underline{3077.91741}$

Partridge, S. R., Juan, S. J., McGeechan, K., Bauman, A., \& Allman-Farinelli, M. (2015). Poor quality of external validity reporting limits generalizability of overweight and/or obesity lifestyle prevention interventions in young adults: a systematic review. Obesity Reviews, 16(1), 13-31. https://doi.org/10.1111/obr.12233

Pope, L., Hansen, D., \& Harvey, J. (2016). Examining the Weight Trajectory of College Students. Journal of Nutrition Education and Behavior. https://doi.org/10.1016/j.jneb.2016.10.014

Rust, G. (2017). Choosing Health Equity: Investing in Optimal and Equitable Health for All. American Journal of Public Health, 107(3), 361-363. https://doi.org/10.2105/ AJPH.2016.303645 
Salehi, A. M. M. P., Marzban, M. M., Sourosh, M. P., Sharif, F. P., Nejabat, M. M., \& Imanieh, M. H. M. (2017). Social Well-Being and Related Factors in Students of School of Nursing and Midwifery. International Journal of Community Based Nursing and Midwifery, 5(1), 82-90.

Tempels, T., Verweij, M., \& Blok, V. (2017). Big Food's Ambivalence: Seeking Profit and Responsibility for Health. American Journal of Public Health, 107(3), 402-406. https://doi. org/10.2105/AJPH.2016.303601

Walker, M., Thornton, L., De Choudhury, M., Teevan, J., Bulik, C. M., Levinson, C. A., \& Zerwas, S. (2015). Facebook Use and Disordered Eating in College-Aged Women. Journal of Adolescent Health, 57(2), 157-163. https://doi.org/10.1016/j.jadohealth.2015.04.026

Zimmerman, B. J. (1989). A social cognitive view of self-regulated academic learning. Journal of Educational Psychology, 81(3), 329-339. https://doi.org/10.1037/0022-0663.81.3.329

1 Yolanda Campos-Uscanga .Licenciada en Psicología, Maestra en Salud Pública con área disciplinar en Epidemiología y Doctora en Psicología. Investigadora de tiempo completo del Instituto de Salud Pública, Universidad Veracruzana, Xalapa, Veracruz, México.

2 Tania Romo-González. Química Farmacéutico Bióloga y Doctora en Investigación Biomédica Básica. Investigadora de tiempo completo del Área de Biología y Salud Integral, Instituto de Investigaciones Biológicas, Universidad Veracruzana, Xalapa, Veracruz, México.

3 Luis Eduardo del Moral-Trinidad. Estudiante de la Licenciatura en Medicina, Universidad Veracruzana, Xalapa, Veracruz, México.

3 Nelly Itzel Carmona-Hernández. Estudiante de la Licenciatura en Medicina, Universidad Veracruzana, Xalapa, Veracruz, México 